\title{
The $\mathfrak{s o}(d+2,2)$ minimal
}

\section{representation and ambient}

\section{tractors: the conformal geometry of momentum space}

\author{
Ashwin Rod Gover ${ }^{1}$ and Andrew Waldron ${ }^{2}$ \\ ${ }^{1}$ Department of Mathematics, The University of Auckland, Auckland \\ PB 1142, New Zealand \\ gover@math. auckland.ac.nz \\ ${ }^{2}$ Department of Mathematics, University of California, Davis, \\ CA 95616, USA \\ wally@math.ucdavis.edu
}

\begin{abstract}
Tractor calculus is a powerful tool for analyzing Weyl invariance; although fundamentally linked to the Cartan connection, it may also be arrived at geometrically by viewing a conformal manifold as the space of null rays in a Lorentzian ambient space. For dimension $d$ conformally flat manifolds we show that the $(d+2)$-dimensional Fefferman-Graham ambient space corresponds to the momentum space of a massless scalar field. Hence on the one hand the null cone parameterizes physical momentum excitations, while on the other hand, null rays correspond to points in the underlying conformal manifold. This allows us to identify a fundamental set of tractor operators with the generators of conformal symmetries of a scalar field theory in a momentum representation. Moreover,
\end{abstract}

e-print archive: http://lanl.arXiv.org/abs/0903.1394 
these constitute the minimal representation of the non-compact conformal Lie symmetry algebra of the scalar field with Kostant-Kirillov dimension $d+1$. Relaxing the conformally flat requirement, we find that while the conformal Lie algebra of tractor operators is deformed by curvature corrections, higher relations in the enveloping algebra corresponding to the minimal representation persist. We also discuss potential applications of these results to physics and conformal geometry.

\section{Introduction}

The massless wave equation is ubiquitous in both physics and mathematics. In this article we point out yet another rôle for this equation in spaces of metric signature $(p-1, q-1)$ : (in a suitably flat setting) its solution space describes the conformal geometry of pseudo-Riemannian manifolds of signature $(p-2, q-2)$. The key idea is that solutions to the flat space wave equations on $\mathbb{R}^{p-1, q-1}$ are parameterized by light-like momenta. In this picture the space of all possible "off-shell" momenta corresponds to the ambient space of a $d=(p+q-4)$-dimensional conformal manifold; the latter can be viewed as the space of light-like rays in a certain null hypersurface. Although our results hold in any signature $p, q>1$ (with $p+q \geq 5$ ), we set $(p, q)=$ $(d+2,2)$ for simplicity of exposition; we leave it as an exercise for the reader to make the minor adjustments to recover other signatures. We will call the space $\mathbb{R}^{d+1,1}$ hosting the scalar wave equation the "dual ambient space."

The duality that we study in this paper is defined via Fourier transform. An important feature of this construction are the symmetries following from the dual ambient conformal group $\mathrm{SO}(d+2,2)$. It is well known that solutions to the scalar wave equation form an irreducible unitary representation of this group. In fact, a recent study by Kobayashi and Ørsted [1] gives an explicit construction of the intertwiner by Fourier transform of the reducible $\mathrm{SO}(d+2,2)$ representation on the dual ambient space. The restriction of which recovers an irreducible one on the ambient lightcone and they identify it (for $d \geq 4$ ) as the minimal representation (see $[2,3]$ ). This idea of studying conformal symmetry by looking at spaces of two higher dimensions dates back to Dirac [4], but the additional Fourier duality gives a great deal of geometric information.

In this article, the differential generators of $\mathfrak{s o}(d+2,2)$ acting on the dual ambient space will be shown to play a distinguished geometric rôle once intertwined to act on the ambient lightcone. The relevant geometry is described by the tractor calculus for conformal manifolds of $[5,6]$. Central to the tractor calculus, is a basic set of differential operators that were 
constructed at first in an apparently ad hoc way in [5-8]. In fact, these are strongly linked to representation theory and collectively arise from the fundamental derivative found in [9]; the latter is a first-order differential operator with universal properties, existing on a large range of structures. Later, these tractor operators were recovered $[10,11]$ from the Fefferman-Graham (FG) ambient manifold [12] associated canonically with any conformal manifold (with dimension greater than two). Here we show that, at least in the conformally flat case, the collection of tractor operators is unified and arises independently from the picture above. In fact, they are the differential generators of the minimal representation on the lightcone. Therefore, from this point of view, their origin is as the generators of the dual ambient conformal group. This relationship is expressed by the intertwiner by Fourier transform. It follows that, in the conformally flat setting, the tractor operators (see (4.8)) obey an $\mathfrak{s o}(d+2,2)$ algebra (see (4.4)). Moreover, the underlying minimal representation carries further geometric information in the form of quadratic relations among tractor operators. Returning to generally curved conformal manifolds, the $\mathfrak{s o}(d+2,2)$ Lie algebra structure is deformed to reflect the curvature of the ambient manifold. Remarkably, we show that these quadratic relations continue to hold, and encode the key identities obeyed by the tractor operators themselves. Although these identities were known previously, this new derivation provides a structure explaining why these identities exist and suggests an obvious extension to other geometric structures.

The remainder of this article is organized as follows. We begin by giving some key facts about tractors and review the FG ambient metric construction for a conformal manifold in Sections 2 and 3. Then we introduce the massless scalar field theory and its conformal symmetries in Section 4. In Section 5 we establish the relationship between tractor operators and the ambient conformal symmetries. In Section 6 we show how to generalize our results to curved ambient spaces and conformal manifolds that are not conformally flat. Potential applications to both physics and conformal geometry are discussed in the conclusions.

\section{Tractors}

Weyl transformations

$$
g_{\mu \nu} \longmapsto \hat{g}_{\mu \nu}=\Omega^{2} g_{\mu \nu}
$$

are fundamental to both physics and mathematics. In particular, conformal geometry is the study of manifolds equipped with a (conformal) equivalence 
class of metrics where the equivalence relation is given by $g \sim \hat{g}$ if $g$ and $\hat{g}$ are related as in (2.1), and where $\Omega$ is any non-vanishing function on the given $d$-dimensional manifold $M$.

In pseudo-Riemannian geometry the standard invariant calculus is based around the Levi Civita connection $\nabla_{\mu}$ determined canonically by the metric $g_{\mu \nu}$. The fundamental local invariant is the Riemannian curvature $R_{\mu \nu}{ }_{n}{ }_{n}$ and we recall that this decomposes according to

$$
R_{\mu \nu \rho \sigma}-W_{\mu \nu \rho \sigma}=\mathrm{P}_{\mu \rho} g_{\nu \sigma}-\mathrm{P}_{\nu \rho} g_{\mu \sigma}-\mathrm{P}_{\mu \sigma} g_{\nu \rho}+\mathrm{P}_{\nu \sigma} g_{\mu \rho},
$$

where $W$ is the completely trace-free part of the curvature $R$ and $\mathrm{P}$ is the Schouten tensor. Descending to the equivalence class of metrics of conformal geometry there is no satisfactory conformally invariant connection on the tangent bundle and this frustrates naïve attempts to construct well-defined natural equations and invariants. On the other hand, in dimensions $d \geq 3$ (and we make this restriction throughout) there is a conformally invariant connection on a bundle of rank $d+2$ which extends the tangent bundle. In a metric scale this (standard) tractor bundle, denoted $\mathcal{E}^{M}$ (in a Penrose notation), is simply a direct sum of the tangent bundle with two copies of the trivial bundle and the tractor connection is given explicitly by

$$
\mathcal{D}_{\mu}\left(\begin{array}{c}
T^{+} \\
T^{m} \\
T^{-}
\end{array}\right)=\left(\begin{array}{c}
\nabla_{\mu} T^{+}-T_{\mu} \\
\nabla_{\mu} T^{m}+\mathrm{P}_{\mu}{ }^{m} T^{+}+e_{\mu}{ }^{m} T^{-} \\
\nabla_{\mu} T^{-}-\mathrm{P}_{\mu n} T^{n}
\end{array}\right),
$$

where $T^{+}$and $T^{-}$are functions while $T^{m}$ is a tangent vector field. This connection is equivalent to the normal Cartan connection of [13], see [9], but the first construction and use of it as here was (independently) by Thomas [5].

The connection $\mathcal{D}_{\mu}$ preserves a conformally invariant tractor metric $h_{M N}$ (of signature $(d+1,1)$ for Riemannian conformal geometry) which is given as a quadratric form on $T^{M}$ by the formula $h_{M N} T^{M} T^{N}=2 T^{+} T^{-}+T^{m} T_{m}$. Thus we see some analogies with the pseudo-Riemannian calculus. However observe that the tractor covariant derivative of $T^{M}$, viz. $\mathcal{D}_{\mu} T^{M}$ is a mixed one-form-tractor and so the route to higher derivatives, captured in some conformally invariant way, is not immediately clear. Solving this in general turns out to be rather subtle and this is one of the key applications of tractor calculus. This point of view has its origins in [5] and was rediscovered and developed significantly in [6,8-11]. It first appeared in a physics context in the supergravity-motivated study of conformal gravity [14] and these ideas have also been applied recently to show how Weyl invariance underlies the origins of mass in particle equations $[15,16]$. Among these $[6,10,11,15]$, for example, give background for details of the calculations described. 
Part of the solution, to the alluded problem, involves a collection of conformally invariant differential operators that can be iterated without moving out of the tractor framework and these are the subject of the current article. Before we describe these, some notation is required. We will often work with tractor bundles with (or "twisted by") a conformal weight which may be denoted $w$. We write $\mathcal{E}^{M}[w]$ for the weight $w$ tractor bundle. Weighted tensor powers of the standard tractor bundle are denoted $\mathcal{E}^{M N \ldots}[w]$ and accordingly with $(\cdots)$ and $[\cdots]$ denoting symmetrization and antisymmetrization, respectively. So with this notation the key tractor-valued operators of our focus act as follows:

$$
\begin{aligned}
X^{M}: \mathcal{E}^{N \cdots}[w] & \longrightarrow \mathcal{E}^{M N \cdots}[w+1], \\
D^{M N}: \mathcal{E}^{R \cdots}[w] & \longrightarrow \mathcal{E}^{[M N] R \cdots[w]} \\
D^{M}: \mathcal{E}^{N \cdots}[w] & \longrightarrow \mathcal{E}^{M N \cdots}[w-1] .
\end{aligned}
$$

The first of these is defined by multiplication by the canonical tractor $X^{M}$. The Thomas- $D$-operator $D^{M}$ and double- $D$ operator $D^{M N}$ are defined in the standard way. To this set of tractor operators, it will be important to add the operator whose eigenvectors are tractors of definite weight and eigenvalue is the weight. The operators in (2.3) can also be expressed explicitly in terms of the tractor connection for a given choice of metric as

$$
\begin{gathered}
X^{M}=\left(\begin{array}{l}
0 \\
0 \\
1
\end{array}\right), \quad D^{M N}=\left(\begin{array}{ccc}
0 & 0 & w \\
0 & 0 & \mathcal{D}^{m} \\
-w & -\mathcal{D}^{n} & 0
\end{array}\right), \\
D^{M}=\left(\begin{array}{c}
w(d+2 w-2) \\
(d+2 w-2) \mathcal{D}^{m} \\
-\left(\mathcal{D}^{r} \mathcal{D}_{r}+w \mathrm{P}\right)
\end{array}\right) .
\end{gathered}
$$

That they map objects covariant under tractor gauge transformations to objects with the same covariance can be checked by explicit calculation but also follows from general principles [6]. More importantly, their extensive practical applicability to constructing Weyl invariants and covariants, as well as physical theories from first principles, is clear $[8,11,15-17]$.

\section{The conformal ambient metric construction}

A useful way to view a $d$-dimensional conformal manifold $\left(M,\left[g_{\mu \nu}\right]\right)$ is as the space of rays in a $(d+1)$-dimensional null hypersurface $Q$ in a $(d+2)$-dimensional Riemannian ambient space $\left(\tilde{M}, h_{M N}\right)$. More 


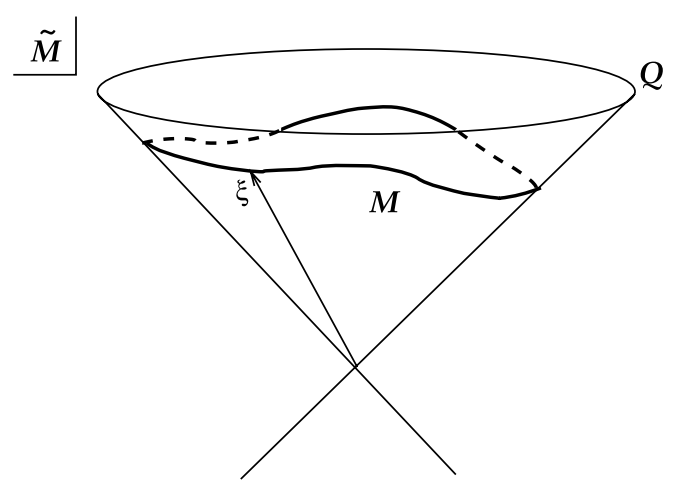

Figure 1: The sphere realized as rays $\xi$ of the cone $Q$ in the ambient space $\tilde{M}$.

precisely — specializing to the conformally flat case - consider the ambient space $\tilde{M}=\mathbb{R}^{d+1,1}$ with the standard flat Lorentzian metric

$$
\tilde{d} s^{2}=d X^{M} \eta_{M N} d X^{N} .
$$

This metric enjoys a hypersurface orthogonal homothety given by the Euler operator $^{1}$

$$
\mathbf{w}=X^{M} \frac{\partial}{\partial X^{M}}, \quad £_{\mathbf{w}} \tilde{d} s^{2}=2 \tilde{d} s^{2} .
$$

The zero locus of the homothetic potential

$$
H=\frac{1}{2} X^{M} X_{M} \equiv \frac{1}{2} X^{2}
$$

defines the null cone $Q$. $H$ is the defining function for the cone $Q$. The space of null rays in $Q$, viz., solutions $\xi^{M}$ to $H=0$ subject to the equivalence relation

$$
\xi^{M} \sim \Omega \xi^{M}, \quad \Omega \in \mathbb{R}^{+}
$$

is $d$-dimensional and may be identified with the conformal manifold $M$. This is depicted in figure 1 . We may obtain the conformal class of metrics as follows: Let $\xi^{M}(x)$ denote a section of the null cone $Q$. The ambient metric pulls back to a metric $d s^{2}=d \xi^{M} d \xi_{M}$ on the submanifold. Identifying this submanifold with $M$ yields a metric $d s^{2}$ on $M$. Choosing a different section results in a conformally related metric. For example, in the conformally flat setting, the sphere, flat and hyperbolic metrics all inhabit the

\footnotetext{
${ }^{1}$ We use $£$ to denote the Lie derivative.
} 


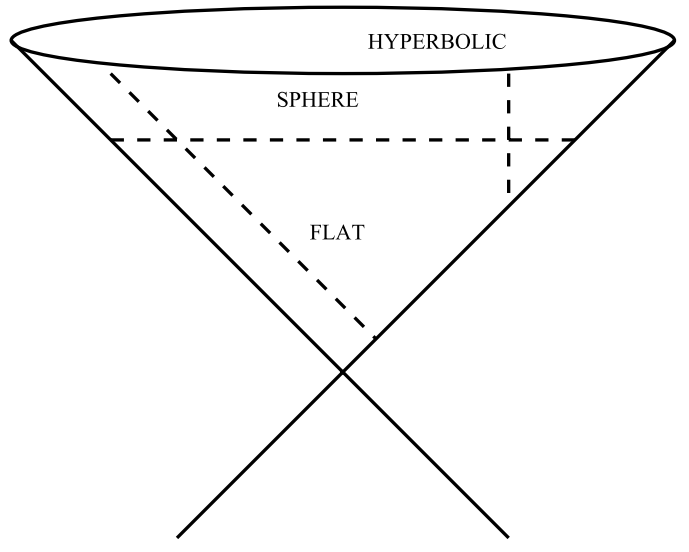

Figure 2: The sphere, flat space and hyperbolic space obtained from slices of the cone $Q$.

same conformal class. In the ambient picture they correspond to slicing the cone horizontally, diagonally and vertically, respectively. This is depicted in figure 2 .

We will obtain the tractor operators in this ambient picture by reinterpreting the null cone $Q$ as the space of massless excitations for a scalar field. In more mundane language, we propose interpreting $Q$ as the onshell lightcone for a scalar field. In particular, in this picture, the ambient coordinates $X^{M}$ must be viewed as momenta. Before studying scalar fields, however, let us briefly indicate the ambient origin of the tractor connection $\mathcal{D}_{\mu}$ : Firstly, observe that the Lorentz group $G=\mathrm{SO}_{o}(d+1,1)$ acts naturally on the ambient space $\tilde{M}=\mathbb{R}^{d+1,1}$. Then pick any future light-like ray $\xi$ in $\tilde{M}$ and call its stabilizer $P$. The coset $G / P$ is exactly the sphere $M$. Indeed, this is the classical model for the sphere equipped with a conformally flat structure. The Maurer-Cartan form on $G$ is $\mathfrak{s o}(d+1,1)$-valued so when pulled back from $G$ to $M=G / P$, it defines a $\mathfrak{s o}(d+1,1)$ Yang-Mills connection. This connection is precisely the tractor connection. Its equivalent parallel transport is that arising from ambient pseudo-Euclidean parallel transport.

\section{Ambient scalar fields}

Unitary representations for non-compact forms of orthogonal groups are necessarily infinite dimensional. There is, however, a notion of Gel'fand-Kirillov dimension $[1,3]$ associated with the number of independent variables for unitary representations on function spaces and in turn representations that are 
in this sense minimal [18]. For orthogonal groups $O(p, q)$, the minimal unitary representation is shown to be related to the space of zero modes of the ultrahyperbolic Laplace operator on $\mathbb{R}^{p-1, q-1}[1,3]$. This setting is of course a familiar one in physics, namely a free massless scalar field in a flat background - essentially the simplest quantum field theory possible. Here we sketch physical aspects of this construction of the minimal unitary representation of $O(d+2,2)$ in order to develop the intuition needed for our construction of a symmetry algebra for tractor operators. (We have chosen this particular signature so that our underlying conformal manifold will be Riemannian. Our results extend immediately to conformal manifolds of any metric signature, however.) Consider therefore a scalar field $\Phi(Y)$ in flat $\mathbb{R}^{d+1,1}$ with local coordinates $Y^{M}$. For reasons which will become clear, we call this space the dual ambient space. Let the dynamics of this scalar field be governed by the action principle

$$
S=-\frac{1}{2} \int d^{d+2} Y \frac{\partial \Phi}{\partial Y^{M}} \eta^{M N} \frac{\partial \Phi}{\partial Y^{N}} .
$$

This action is invariant under $\mathfrak{s o}(d+2,2)$ rigid symmetry transformations corresponding to the conformal Killing vectors

$$
\begin{gathered}
\mathrm{i} P_{M} \equiv \frac{\partial}{\partial Y^{M}} \quad \text { (translations) } \\
D \equiv Y^{M} \frac{\partial}{\partial Y^{M}} \quad \text { (dilations) } \quad M_{M N}=Y_{M} \frac{\partial}{\partial Y^{N}}-Y_{N} \frac{\partial}{\partial Y^{M}} \quad \text { (rotations) } \\
\mathrm{i} K_{M}=-2 Y_{M} D+Y^{N} Y_{N} \frac{\partial}{\partial Y^{M}} \quad \text { (conformal boosts). }
\end{gathered}
$$

Invariance of action (4.1) is achieved by transformations of $\Phi$ with a conformal weight $-d / 2$ generated by

$$
\delta_{X} \Phi=£_{X} \Phi+\frac{d}{2} \alpha_{X} \Phi,
$$

where $X$ stands for any of vectors (4) and the function $\alpha_{X}=(d+2)^{-1} \operatorname{div} X$ so that $£_{X} \tilde{d} s^{2}=2 \alpha_{X} \tilde{d} s^{2}$. In particular $\alpha_{D}=1$ and $\alpha_{K_{M}}=2 \mathrm{i} Y_{M}$.

The operators $\delta_{X}$ in (4.3) acting on scalars generate a (reducible) representation of $\mathfrak{s o}(d+2,2)$. Explicitly, the non-vanishing Lie brackets of the above vector fields are

$$
\begin{gathered}
{\left[M_{M N}, M_{R S}\right]=\eta_{N R} M_{M S}-\eta_{M R} M_{N S}-\eta_{N S} M_{M R}+\eta_{M S} M_{N R},} \\
{\left[D, P_{M}\right]=-P_{M}, \quad\left[D, K_{M}\right]=K_{M},}
\end{gathered}
$$




$$
\begin{gathered}
{\left[M_{M N}, P_{R}\right]=2 P_{[M} \eta_{N] R}, \quad\left[M_{M N}, K_{R}\right]=2 K_{[M} \eta_{N] R}} \\
{\left[P_{M}, K_{N}\right]=2 \eta_{M N} D-2 M_{M N} .}
\end{gathered}
$$

Our aim is to relate the operators $£_{X}+\frac{d}{2} \alpha_{X}$ to tractor operators. This involves identifying the generators of the conformal isometry group $\mathrm{SO}(d+$ $2,2)$ of the dual ambient space with tractor operators on an ambient space $\tilde{M}$. However, it is important to already stress that the $\mathbb{R}^{d+1,1}$ dual ambient space on which our scalar field theory lives is not the ambient manifold $\tilde{M}$ but rather will be related to it by a Fourier transformation. To study this relationship we first solve the classical field theory defined by (4.1).

The field equation implied by the action (4.1)

$$
\Delta \Phi=0,
$$

may be solved by Fourier transform

$$
\Phi(Y)=\int \frac{d^{d+2} X \delta\left(X^{2}\right)}{(2 \pi)^{d+2}} \exp \left(\mathrm{i} X_{M} Y^{M}\right) \varphi(X),
$$

whose integrand has delta function support on the light cone $Q$. This mapping between solutions to the ultrahyperbolic wave equation (4.5) and distributions supported on the null cone $Q$ with defining function $H=\frac{1}{2} X^{2}$ is constructed in detail in [1]. Since our main focus is identifying the image of the conformal ambient $\mathfrak{s o}(d+2,2)$ differential generators under the Fourier intertwiner (4.6), we shall avoid a detailed discussion of the function spaces involved. Physically, the set of variables $\left(X_{M}\right)$ is interpreted as an $\mathbb{R}^{d+1,1_{-}}$ valued momentum. We advocate identifying this momentum space with the ambient manifold $\tilde{M}$. By including the $\delta$ distribution explicitly we execute a truncation of $\varphi(X)$. Even though only values of $\varphi(X)$ along the cone are important for the integral, to recover calculus with ambient content we shall impose the condition that smooth $\varphi(X)$ on the cone are also extended off smoothly; that is we take $\varphi(X)$ to be smooth on the ambient space. In particular, the solution space may be viewed as an equivalence class of functions

$$
\varphi(X) \sim \varphi(X)+H \xi(X)
$$

for a suitable class of functions $\xi(X)$. For our purposes, smooth functions suffice.

The Fourier transform (4.6) is an intertwiner between $\mathfrak{s o}(d+2,2)$ representations. Acting on arbitrary functions of $\tilde{M}$ (regardless of their support away from $Q$ ) it is simple to compute the differential representation of 
$\mathfrak{s o}(d+2,2)$ using the usual correspondence under Fourier transforms $Y^{M} \rightarrow$ $\mathrm{i} \partial / \partial X_{M}$ and $\partial / \partial Y^{M} \rightarrow \mathrm{i} X^{M}$. The resulting operators are not vector fields on $\tilde{M}$ (save for the rotations $M_{M N}$ ) but, necessarily, obey the $\mathfrak{s o}(d+2,2)$ Lie algebra. Also, they are not quite the operators we need. Instead we are interested in what becomes of the symmetry operators $£_{X}+\frac{d}{2} \alpha_{X}$ when passed through the intertwiner (4.6), including the delta function $\delta\left(X^{2}\right)$, so that they act on the physical moduli $\varphi(X)$. In this simple flat setting, this computation can be done explicitly by actually performing the delta function integration. To contrast them from their underlying Killing vectors (4), we denote these intertwined symmetry generators in bold face and find the following operators agree with the action of the conformal Killing symmetry operators

$$
\begin{gathered}
\mathbf{P}_{M}=X_{M}, \\
\mathbf{D}=-X^{M} \frac{\partial}{\partial X^{M}}-d / 2, \quad \mathbf{M}_{M N}=X_{M} \frac{\partial}{\partial X^{N}}-X_{N} \frac{\partial}{\partial X^{M}}, \\
\mathbf{K}_{M}=-2 \mathbf{D} \frac{\partial}{\partial X^{M}}-X_{M} \frac{\partial}{\partial X^{N}} \frac{\partial}{\partial X_{N}} .
\end{gathered}
$$

An important consequence of the symmetries (4.3) is that dual ambient space conformal transformations map solutions of (4.5) to solutions. Working to the right of the Dirac distribution (as a multiplication operator) for consistency with our point of view above we require image operators that respect the equivalence relation (4.7). To see this explicitly for the operators proposed, we compute the commutators of the generators (4.8) with the defining function $H$, the non-vanishing results are

$$
[\mathbf{D}, H]=-2 H, \quad\left[\mathbf{K}_{\mathbf{M}}, H\right]=4 H \partial_{M} .
$$

Hence if $\varphi \sim \tilde{\varphi}$ under (4.7), then $\mathbf{X} \varphi \sim \mathbf{X} \tilde{\varphi}$ for $\mathbf{X}$ equaling any of the generators (4.8). This was noted by [11] where it was proven that the operators (4.8) act tangentially to the cone defined by $H$. Here we see that this result enables natural ambient extensions of the Fourier duals to the conformal symmetries of the massless scalar field theory (4.1). Before recasting this physical theory in terms of tractors and conformal geometry, we briefly discuss the minimal representation and some of its consequences.

\subsection{The minimal representation}

Our computation began with off-shell massless scalar fields $\Phi(Y)$ functions of $d+2$ variables - on which the group $\mathrm{SO}(d+2,2)$ acts by conformal transformations (4.3). This is not an irreducible representation 
but instead closely related to one. The Fourier transform (4.6) yields a representation on on-shell massless fields in momentum space given by (4.8). As is well known in a physical setting, this is a unitary and irreducible representation of $\mathrm{SO}(d+2,2)$. Indeed, as shown in [1], this is the minimal representation of $\mathrm{SO}(d+2,2)$ (for $d \geq 4$ ) and the number of variables required to coordinatize the cone $Q$ is its Kostant-Kirillov dimension $d+1$. The Fourier transform is the intertwiner between these representations.

The minimal representation admits a set of quadratic relations in the universal enveloping algebra $\mathcal{U}(\mathfrak{s o}(d+2,2))$ which imply important tractor identities in a conformal geometry setting. One may characterize representations by relations among generators in the universal enveloping algebra. Consider, therefore, the matrix of $\mathfrak{s o}(d+2,2)$ symmetry generators

$$
J=\left(\begin{array}{ccc}
-\mathbf{D} & -\frac{1}{\sqrt{2}} \mathbf{P}_{M} & 0 \\
\frac{1}{\sqrt{2}} \mathbf{K}_{N} & \mathbf{M}_{M N} & \frac{1}{\sqrt{2}} \mathbf{P}_{N} \\
0 & -\frac{1}{\sqrt{2}} \mathbf{K}_{M} & \mathbf{D}
\end{array}\right) .
$$

In particular, notice that

$$
\operatorname{tr} J^{2}=\mathbf{M}_{M N} \mathbf{M}^{N M}+2 \mathbf{D}^{2}-\mathbf{P}^{M} \mathbf{K}_{M}-\mathbf{K}^{M} \mathbf{P}_{M}
$$

is the quadratic Casimir. In fact, for representation (4.8) it obeys

$$
\operatorname{tr} J^{2}=-\frac{1}{2} d(d+4)
$$

a constant, as is necessarily the case for an irreducible representation. However, we can search for even stronger relations by computing $J^{2}$ and find

$$
(J+1)(J+d / 2)=0 \bmod H,
$$

i.e., the right-hand side vanishes on the cone $Q$ in the sense of (4.7). Explicitly, this implies the following relations:

$$
\begin{gathered}
\mathbf{P}_{M} \mathbf{P}^{M}=0 \\
\mathbf{P}_{M}(\mathbf{D}+d / 2)-\mathbf{P}^{N} \mathbf{M}_{N M}=0 \\
-\frac{1}{2} \mathbf{P}_{M} \mathbf{K}_{N}+\mathbf{M}_{M}{ }^{R} \mathbf{M}_{R N}-\frac{1}{2} \mathbf{K}_{M} \mathbf{P}_{N}-(1+d / 2) \mathbf{M}_{M N}+d / 2 \eta_{M N}=0 \\
(\mathbf{D}+1)(\mathbf{D}+d / 2)-\frac{1}{2} \mathbf{P}_{N} \mathbf{K}^{N}=0 \\
(\mathbf{D}+d / 2) \mathbf{K}_{M}-\mathbf{M}_{M N} \mathbf{K}^{N}=0 \\
\mathbf{K}^{M} \mathbf{K}_{M}=0 .
\end{gathered}
$$


These relations all have tractor theoretic interpretations which we will analyze in the next section.

\section{Tractors and the ambient conformal group}

In $[10,11]$ it is shown that the tractor operators (2.3) may be recovered from (curved versions of) the ambient operators (4.8). The latter act on ambient functions $\varphi(X)$. However, if we impose both the equivalence relation (4.7) and the homogeneity condition

$$
X^{M} \frac{\partial}{\partial X^{M}} \varphi=(-\mathbf{D}-d / 2) \varphi=w \varphi,
$$

it follows that $\varphi$ is a section of $\mathcal{E}[w]$, i.e., a weight $w$ scalar field on $M$. Moreover, since the ambient operators (4.8) are homogeneous and tangential, they descend to well-defined operators acting on weighted tractor bundles over $M$, as listed in (2.3). We refer to [11] for details where it is also shown, in an obvious way, that the same property extends to arbitrary tractor tensors.

Therefore, in the conformally flat context, we have established that the tractor operators are the generators of the dual ambient conformal group $\mathfrak{s o}(d+2,2)$ in a momentum representation. This dictionary between geometry and physics is summarized in Table 1.

Next, we explain how to read the minimal representation theoretic relations (4.12) as relations on tractor identities. Firstly, the canonical tractor $X^{M}$ and Thomas operator $D^{M}$ are both null. Let us suppose these operators are acting on functions of conformal weight $w$. Therefore $\mathbf{D}$ acts as multiplication by $-w-\frac{d}{2}$ and the Thomas operator becomes

$$
D_{M}=(d+2 w-2) \partial_{M}-X_{M} \partial_{N} \partial^{N},
$$

Table 1: Geometry-physics dictionary.

\begin{tabular}{ccccc}
\hline \multicolumn{2}{c}{ Physics } & & & \multicolumn{2}{c}{ Mathematics } \\
\cline { 1 - 2 } \cline { 5 - 5 } Operator & Symbol & & Operator & Symbol \\
\hline Momentum & $\mathbf{P}_{M}$ & & Canonical tractor & $X_{M}$ \\
Dilations & $\mathbf{D}$ & & Weight & $-w-d / 2$ \\
Rotations & $\mathbf{M}_{M N}$ & & Double- $D$-operator & $D_{M N}$ \\
Conformal boosts & $\mathbf{K}_{M}$ & & Thomas $D$-operator & $D_{M}$ \\
\hline
\end{tabular}


in agreement with the results of [11]. Moreover it follows from (4.12) that the top slot of the Thomas operator is given by

$$
X_{M} D^{M}=w(d+2 w-2),
$$

which agrees with (2.3). From the third identity in (4.12) we recover the formula relating the product of double- $D$ operators to the Thomas operator itself $[7,8]$

$$
D_{(M}^{R} D_{|R| N)_{0}}=X_{(M} D_{N)_{0}},
$$

where $(\cdots)_{0}$ denotes trace-free symmetrization with unit weight. Other simple yet useful identities, which all follow from (4.12) include

$$
X^{N} D_{M N}=w X_{M}, \quad D_{M N} D^{N}=-(w-1) D_{M} .
$$

Moreover, it should be kept in mind that the operators $\left\{\mathbf{P}_{\mathbf{M}}, \mathbf{D}, \mathbf{M}_{\mathbf{M N}}, \mathbf{K}_{\mathbf{M}}\right\}$ only obey the $\mathfrak{s o}(d+2,2)$ Lie algebra (4.4) when the ambient space is flat. For curved ambient spaces and therefore general conformal manifolds, this is no longer the case. This is the subject of the next section.

\section{Curved ambient spaces}

In this section we sketch the curved ambient construction of the tractor operators $[10,11]$ generalizing those above. We will then show that although a non-vanishing ambient curvature deforms the $\mathfrak{s o}(d+2,2)$ algebra obeyed in the flat ambient case, the quadratic relations (4.12) of the minimal representation persist.

A conformal structure on $M$ determines a FG ambient metric which admits a hypersurface orthogonal homothety. In the flat case this homothety is generated by the Euler vector field. In the curved ambient construction, the corresponding homothetic vector field will be denoted by $X$ and its components $X^{M}$ (which are not generally coordinates). The key identity is then the equation

$$
h_{M N}=\nabla_{M} X_{N},
$$

where $h$ is the ambient metric and $\nabla$ is its Levi-Civita covariant derivative. This implies the homothetic conformal Killing equation

$$
£_{X} h=2 h .
$$

It follows that the one-form dual to $X$ is closed

$$
\nabla_{[M} X_{N]}=0,
$$


and in fact exact

$$
X_{M}=\frac{1}{2} \nabla_{M} X^{2} .
$$

Clearly, the ambient metric is the double gradient of the homothetic potential $H=\frac{1}{2} X^{2}$,

$$
h_{M N}=\nabla_{M} \partial_{N} H .
$$

The zero locus of the potential $H$ defines the cone $Q$, a quotient of which recovers the conformal manifold $M$. Observe that the above identities for the ambient metric $h_{M N}$ imply that

$$
X^{M} R_{M N R S}=0=\left(X^{T} \nabla_{T}+2\right) R_{M N}{ }_{S} .
$$

To establish a Rosetta Stone between ambient space operators and the tractor operators (2.3) on the conformal manifold, our prescription is to first replace partial derivatives by covariant ones and coordinates by the components of the homothety $X$ (and then use [10]). This gives the following dictionary for curved ambient generalizations of the conformal generators:

$$
\begin{aligned}
& \mathbf{M}_{M N} \longmapsto D_{M N} \equiv 2 X_{[M} \nabla_{N]}, \\
& \mathbf{P}_{M} \longmapsto X_{M}, \quad \mathbf{K}_{M} \longmapsto D_{M} \equiv 2\left(X^{N} \nabla_{N}+d / 2\right) \nabla_{M}-X_{M} \Delta, \\
& \mathbf{D} \longmapsto-X^{N} \nabla_{N}-d / 2 .
\end{aligned}
$$

Here, just as in (2.3), $X_{M}$ acts as a multiplication operator. Importantly, these operators act on sections of the tensor bundle on $\tilde{M}$.

These operators are rather remarkable in the sense that the quadratic relations (4.12) imposed on $\mathcal{U}(\mathfrak{s o}(d+2,2))$ by the minimal representation are still valid even though they do not, in general, obey the $\mathfrak{s o}(d+2,2)$ dual ambient conformal algebra. To see this, we first note that by virtue of the definition of the ambient metric, the symbols $X_{M}, \nabla_{M}$ and $h_{M N}$ obey the same algebra as their flat counterparts $X_{M}$ (viewed as coordinates), $\partial_{M}$ and $\eta_{M N}$

$$
\left[\nabla_{M}, X_{N}\right]=h_{M N}
$$

except that covariant derivatives no longer commute

$$
\nabla_{[M} \nabla_{N]}=\frac{1}{2} R_{M N}^{\sharp} .
$$


The multiplicative operator $R_{M N}^{\sharp}$ denotes the usual matrix action of the Riemann tensor (for example $R_{M N}^{\sharp} V^{R}=R_{M N}{ }_{S} V^{S}$ ).

Now, using the identity $X^{M} R_{M N}^{\sharp}=0$, it is not difficult to verify that all the quadratic relations in (4.12) hold modulo $X^{2}$. Moreover, the quadratic Casimir continues to obey (4.10). That leaves us only deformations of the Lie algebra itself, the non-vanishing ones are

$$
\begin{aligned}
{\left[D_{M N}, D_{R S}\right]-4 h_{[N[R} D_{M] S]}=} & 4 X_{[R} X_{[M} R_{N] S]}^{\sharp}, \\
{\left[D_{M N}, D_{R}\right]-2 h_{R[N} D_{M]}=} & 2 X_{R} R_{M N}^{\sharp}+2 X_{[M} R_{N] R}^{\sharp}(d+2 X . \nabla-2) \\
& -2 X_{R} X_{[M}\left(R_{N] S}^{\sharp} \nabla^{S}+\nabla^{S} R_{N] S}^{\sharp}\right), \\
{\left[D_{M}, D_{N}\right]=} & \left(R_{M N}^{\sharp}(d+2 X . \nabla-4)+2 X_{[M}\left(\nabla^{R} R_{N] R}^{\sharp}\right.\right. \\
& \left.\left.+R_{N] R}^{\sharp} \nabla^{R}\right)\right)(d+2 X . \nabla-2) .
\end{aligned}
$$

(Here the right-hand sides should be read as operator expressions and $X . \nabla \equiv$ $X^{N} \nabla_{N}$.) Again, these relations are computed modulo $X^{2}$, while tensors of a given weight $w$ on $M$ correspond to eigenfunctions of $X$. $\nabla$ with eigenvalue $w$. In particular, note that Thomas-D operators commute on objects with weight $w=1-d / 2$, in accordance with its interpretation as the Yamabe operator in that case (see (2.3)).

Since the left-hand sides of (6.3) are tangential, so too are the right-hand sides, although this is not manifest in the form given above. To manifest tangentiality consider, for example, the third formula (which was previously given in $[11,19])$ and firstly replace $X . \nabla$ with $w$ acting on weight $w$ tensors. Moreover, using (6.2) and (6.1) it follows that $(d+2 w-2) R_{M N}^{\sharp} \nabla^{N}=$ $R_{M N}^{\sharp} D^{N}$. Furthermore, the FG ambient metric is formally Ricci flat in any odd dimension (to all orders), and Ricci flat to finite order in the defining function $H$ in even dimensions greater than four. Orchestrating these facts we obtain the simpler rewriting of the commutator of Thomas- $D$ operators

$$
\left[D_{M}, D_{N}\right]=(d+2 w-4) R_{M N}^{\sharp}+4 X_{[M} R_{N] R}^{\sharp} D^{R} .
$$

This identity is valid in dimensions other than four and is fundamental in the sense that the other tractor commutators can be derived from it.

To summarize, we have recovered the usual curved tractor algebra along with a new interpretation in terms of the ambient conformal algebra (and 
its deformation). In particular the $\mathfrak{s o}(d+2,2)$ generator-valued matrix

$$
J=\left(\begin{array}{ccc}
w+d / 2 & -\frac{1}{\sqrt{2}} X_{M} & 0 \\
\frac{1}{\sqrt{2}} D_{N} & D_{M N} & \frac{1}{\sqrt{2}} X_{N} \\
0 & -\frac{1}{\sqrt{2}} D_{M} & -w-d / 2
\end{array}\right),
$$

obeys

$$
(J+1)(J+d / 2)=0 \quad \bmod H \quad \text { and } \quad \operatorname{tr} J^{2}=-\frac{1}{2} d(d+4) .
$$

This implies that even though the $\mathfrak{s o}(d+2,2)$ algebra is deformed, the defining relations (4.12) of the minimal representation are maintained.

\section{Conclusions}

In this article we have established a relationship between ambient tractor operators and symmetries of a physical quantum field theory. There are many possible implications. Firstly, in another direction, tractors in their application to Weyl invariance can be used to formulate massive and massless theories within a single Weyl invariant framework $[15,16]$. From this we find that Weyl invariance as a fundamental principle for constructing physical theories yields interesting consequences. In particular, masses turn out to be tractor weights and very general Breitenlohner-Freedman stability bounds $[20,21]$ follow directly. The fusion of that work with the direction of this Article should shed further light on the meaning of mass. In the broader picture our results here point at a relationship between conformal field theories and theories in two higher dimensions, albeit in a dual Fourier transformed space. It is natural to speculate whether such a relationship may capture aspects of the AdS/CFT correspondence [22] as a special case.

From the physical standpoint, our model describes new structure on the geometry of the moduli space of physical excitations for scalar fields. Since the physical lightcone and its accompanying ambient space have a natural curved generalization, one wonders whether this may describe interactions. In particular, renormalization will generate additional momentum dependence of the effective action for an interacting scalar field. From this point of view, therefore, a curved momentum space is most interesting. A study of the map between curved ambient spaces and their underlying configuration space theories is underway [23]. In fact theories with a curved momentum space have been considered before, an example is 
Snyder's non-commutative, yet Lorentz covariant geometry which is based upon a de Sitter momentum space [24]. Interestingly a "Snyderspace," the supersymmetrization of Snyder's model also exists [25], which hints that a "supertractor" theory may be important. Since the gauging of space time algebras technology of [26] also extends to superalgebras, such a theory certainly exists.

There are many obvious extensions and mathematical applications of the results obtained. The tractor operators (4.8) form the basic tools for the theory of conformal invariants developed in [8]; that reference is concerned with constructing conformal invariants of a general conformal manifold. While in low dimensions this gives a near complete answer, significant gaps remain in high even dimensions. The results in this article suggest the exciting possibility that, for dimension $d$ conformal geometry, the invariant theory might in fact be best described via $\mathfrak{s o}(d+2,2)$ representation theory.

Another interesting observation is that generic weight tractors can be extended harmonically away from the cone. From the dual ambient picture, harmonic objects correspond to those with support on the dual ambient lightcone. Aside from being a useful computational trick, for ambient harmonic tractors the Fourier transform yields a symmetric set-up. Informally, (and using delta distributions as integral kernel operators) this is depicted by the correspondences

$$
\begin{aligned}
\operatorname{ker} \Delta_{Y} & \longleftrightarrow \text { coker } X^{2}, \\
\operatorname{coker} Y^{2} & \longleftrightarrow \operatorname{ker} \Delta_{X} .
\end{aligned}
$$

This seems particularly interesting when one remembers that an ambient harmonic extension corresponds to a collar extension off the boundary of a Poincaré-Einstein manifold, which is obviously relevant to the AdS/CFT correspondence.

On the side of extensions, an obvious direction is to exploit the ideas here for other structures. However since a rich picture has emerged from (essentially) the simplest field equations it seems a first step will be to explore the ambient interpretation of other equations on the dual ambient space.

\section{Acknowledgments}

A.R.G is supported by Marsden Grant no. 06-UOA-029. A.W. is indebted to the University of Auckland for its warm hospitality. 


\section{References}

[1] T. Kobayashi and B. Ørsted, Analysis on the minimal representation of $\mathrm{O}(p, q)$. I. Realization via conformal geometry, Adv. Math. 180 (2003), 486; Analysis on the minimal representation of $\mathrm{O}(p, q)$. II. Branching laws, Adv. Math. 180 (2003), 513; Analysis on the minimal representation of $\mathrm{O}(p, q)$. III. Ultrahyperbolic equations on $\mathbb{R}^{p-1, q-1}$, Adv. Math. 180 (2003), 553.

[2] B. Kostant, The vanishing scalar curvature and the minimal unitary representation of $\mathrm{SO}(4,4)$, in 'Operator Algebras, Unitary Representations, Enveloping Algebras, and Invariant Thoery', eds. A. Connes et al., Progress Math. 92 (1990), 85, Birkhäuser Boston, Boston, MA.

[3] B. Binegar and R. Zierau, Unitarization of a singular representation of $\mathrm{SO}(p, q)$, Commun. Math. Phys. 138 (1991), 245.

[4] P. A. M. Dirac, Wave equations in conformal space, Ann. Math. 37 (1936), 429.

[5] T. Y. Thomas, Proc. N. A. S., 12, 352 (1926), The differential invariants of generalized spaces, Cambridge University Press, Cambridge, 1934.

[6] T. N. Bailey, M. G. Eastwood and A. R. Gover, Thomas's structure bundle for conformal, projective and related structures, Rocky Mtn. J. Math. 24 (1994), 1.

[7] A. R. Gover, Aspects of parabolic invariant theory, Rend. Circ. Mat. Palermo (2), Suppl. No. 59 (1999), 25.

[8] A. R. Gover, Invariant theory and calculus for conformal geometries, Adv. Math. 163 (2001), 206.

[9] A. Čap and A. R. Gover, Tractor calculi for parabolic geometries, Trans. Amer. Soc. 354 (2002), 1511.

[10] A. Čap and A. R. Gover, Standard tractors and the conformal ambient metric construction, Ann. Glob. Anal. Geom. 24 (2003), 231.

[11] A. R. Gover and L. J. Peterson, Conformally invariant powers of the Laplacian, Q-curvature, and tractor calculus, Commun. Math. Phys. 235 (2003), 339.

[12] C. Fefferman and C. R. Graham, Conformal invariants, Elie Cartan et les Mathematiques d'Aujourdhui, Asterique, 1985, 95.

[13] E. Cartan, Les espaces à connexions conformes, Ann. Soc. Pol. Math 2 (1923), 171.

[14] M. Kaku, P. K. Townsend and P. van Nieuwenhuizen, Gauge theory of the conformal and superconformal group, Phys. Lett. B 69 (1977), 304; Properties of conformal supergravity, Phys. Rev. D 17 (1978), 3179. 
[15] A. R. Gover, A. Shaukat and A. Waldron, Nucl. Phys. B 812 (2009), 424 [arXiv:0810.2867 [hep-th]].

[16] A. R. Gover, A. Shaukat and A. Waldron, Weyl invariance and the origins of mass, Phys. Lett. B 675 (2009), 93-97, arXiv:0812.3364 [hepth].

[17] T. Branson and A. R. Gover, Conformally invariant operators, differential forms, cohomology and a generalisation of Q-curvature, Comm. Partial Differential Equations 30 (2005), 1611.

[18] A. Joseph, Minimal realizations and spectrum generating algebras, Comm. Math. Phys. 36 (1974), 325; The minimal orbit in a simple Lie algebra and its associated maximal ideal, Ann. Scient. Ecole Normale Sup. 4éme série 9 (1976), 1.

[19] A. R. Gover and J. Silhan, The conformal Killing equation on formsprolongations and applications, Differential Geom. Appl. 26 (2008), 244 [arXiv:math/0601751].

[20] P. Breitenlohner and D. Z. Freedman, Stability in Gauged extended supergravity, Ann. Phys. 144 (1982), 249; Positive energy in anti-de sitter backgrounds and Gauged extended supergravity, Phys. Lett. B 115 (1982), 197.

[21] L. Mezincescu and P. K. Townsend, Stability at a local maximum in higher dimensional anti-de sitter space and applications to supergravity, Ann. Phys. 160 (1985), 406.

[22] J. M. Maldacena, The large $N$ limit of superconformal field theories and supergravity, Adv. Theor. Math. Phys. 2 (1998), 231 [Int. J. Theor. Phys. 38 (1999), 1113] [arXiv:hep-th/9711200]; E. Witten, Anti-de sitter space and holography, Adv. Theor. Math. Phys. 2 (1998), 253 [arXiv:hep-th/9802150]; S. S. Gubser, I. R. Klebanov and A. M. Polyakov, Gauge theory correlators from non-critical string theory, Phys. Lett. B 428 (1998), 105 [arXiv:hep-th/9802109]; O. Aharony, S. S. Gubser, J. M. Maldacena, H. Ooguri and Y. Oz, Large $N$ field theories, string theory and gravity, Phys. Rept. 323 (2000), 183 [arXiv: hep-th/9905111].

[23] A. Shaukat and A. Waldron, Weyl's Gauge invariance: conformal geometry, spinors, supersymmetry, and interactions, Nucl. Phys. B 829 (2010), 28-47, arXiv:0911.2477.

[24] H. Snyder, Quantized space-time, Phys. Rev. 71 (1947), 38; The electromagnetic field in quantized space-time, Phys. Rev. 72 (1947), 68.

[25] M. Hatsuda and W. Siegel, Snyderspace, Nucl. Phys. B 681 (2004), 152. 
[26] S. Ferrara, M. Kaku, P. K. Townsend and P. van Nieuwenhuizen, Gauging the graded conformal group with unitary internal symmetries, Nucl. Phys. B 129 (1977), 125; P. K. Townsend and P. van Nieuwenhuizen, Simplifications of conformal supergravity, Phys. Rev. D 19 (1979), 3166; P. van Nieuwenhuizen, From SU(3) to Gravity, Fortschritt für Y. Ne'eman, Gotsman and Tauber, Cambridge University Press, 1985; in 'Quantum Groups and their Applications in Physics', Varenna Lectures, 1994, eds. L. Castellani and J. Wess, IOS Press 1996. 